\title{
On the Order of Certain Elements of $J(X)$ and the Adams Conjecture
}

By

\author{
Akira Kono*
}

\section{§1. Introduction}

The Adams conjecture [2] was proved by several mathematicians in different methods (cf. [7], [8], [9], [10], [14], [15] and [19]). But in their methods, the localization plays an important role and so we cannot estimate the order of an element

$$
J \circ\left(\psi^{k}-1\right)(x) .
$$

Let $\eta_{n}$ be the canonical (complex) line bundle over $C P^{n}$ and $k$ an integer. Let $m(n, k)$ be the minimal positive integer such that

$$
k^{m(n, k)} J \circ\left(\psi^{k}-1\right)\left(\eta_{n}\right)=0,
$$

which exists by the Adams conjecture for complex line bundles [2]. We put

$$
e(n, k)=m([n / 2], k) \text {. }
$$

Then the purpose of this paper is to show

Theorem 1. If $X$ is an n-dimensional $C W$ complex, then

$$
k^{e(n, k)} J \circ\left(\psi^{k}-1\right)(x)=0
$$

for any $x \in K(X)$.

On the other hand let

$$
e^{\prime}(n, k)= \begin{cases}e(n, k) & \text { if } k \text { is odd } \\ e(n, k)+1 & \text { if } k \text { is even }\end{cases}
$$

Communicated by N. Shimada, August 2, 1980.

* Department of Mathematics, Kyoto University, Kyoto 606, Japan.

This research was partially supported by the Grant-in-Aid for Scientific Research, (No. 574023), Ministry of Education. 
Then by a quite similar method, we have

Theorem 2. If $X$ is an $n$-dimensional $C W$ complex, then

$$
k^{e^{\prime}(n, k)} J \circ\left(\psi^{k}-1\right)(x)=0
$$

for any element $x \in K O(X)$.

Tu prove the above theorems, we do not use the Adams conjecture for general vector bundles. So as a corollary of Theorem 2, the Adams conjecture is proved. The proof of the above theorems is similar to the proof of the Adams conjecture of Nishida [14] and Hashimoto [10]. But we use relations between the induction homomorphisms and the Adams operations in [12] instead of the localization. We also use the cellular approximation of the Becker-Gottlieb transfer used by Sigrist and Suter in [18] instead of the usual Becker-Gottlieb transfer [8].

The paper is organized as follows: In Section 2 some properties of the Becker-Gottlieb transfer are reviewed. Theorem 1 and Theorem 2 are proved in Section 3 and Section 4 respectively. A property of the real induction homomorphism used in this paper is proved in Appendix.

By a quite similar method to the proof of Theorem 1, we can prove Theorem 1 of Sigrist and Suter [18].

\section{§2. Properties of the Becker-Gottlieb Transfer}

In this section $X$ is an $n$-dimensional finite cell complex, $\boldsymbol{G}$ is a compact Lie group and $\mathbb{H}$ is a closed subgroup of $\boldsymbol{G}$. Let $E$ be the total space of a principal $\boldsymbol{G}$-bundle over $X$. Then $p: E / \boldsymbol{H} \rightarrow X$ is a fibre bundle whose fibre is a compact smooth manifold $\boldsymbol{G} / \boldsymbol{H}$ and whose structure group is a compact Lie group $\boldsymbol{G}$ acting smoothly on $\boldsymbol{G} / \boldsymbol{H}$. Let $t(p):(E / \boldsymbol{H})_{+} \rightarrow X_{+}$be the s-map defined by Becker and Gottlieb in [8]. Since $X_{+}$and $(E / H)_{+}$are finite complexes, $t(p)$ is represented by a map

$$
t: \Sigma^{l} \wedge X_{+} \longrightarrow \Sigma^{l} \wedge(E / H)_{+}
$$

for some $l$. Let $(E / \boldsymbol{H})^{(n)}$ be the $n$-skelton of $E / \boldsymbol{H}$ (for some cellular decomposition) and $j:(E / H)^{(n)} \subset E / \mathbb{H}$ be the inclusion. Then by the cellular approximation theorem, there is a map

$$
t^{\prime}: \Sigma^{l} \wedge X_{+} \longrightarrow \Sigma^{l} \wedge\left((E / H)^{(n)}\right)_{+}
$$

such that 


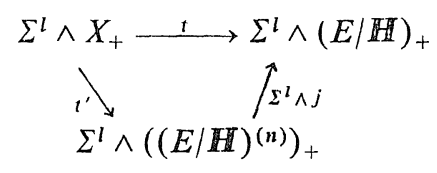

commutes up to homotopy. Define $p_{!}^{\prime}$ by the commutative diagram:

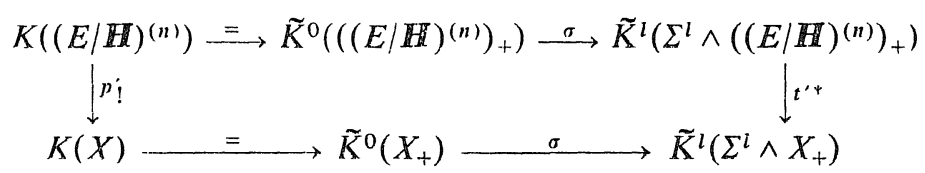

where $\sigma$ is the suspension isomorphism defined by the Bott periodicity theorem ([4]). The Becker-Gottlieb transfer $p_{!}: K(E) \rightarrow K(X)$ is defined by a similar way. Then by definitions the following diagram is commutative:

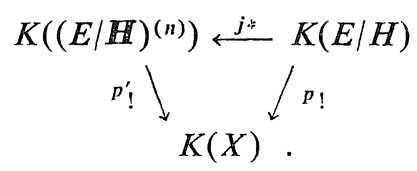

Let $V$ be a complex $\mathbb{H}$-module and $\alpha: R(\mathbb{H}) \rightarrow K(E / \mathbb{H})$ be a homomorphism defined by $V \rightarrow\left(E \times{ }_{\boldsymbol{H}} V \rightarrow E / H\right)$. Define

$$
\alpha^{\prime}: R(\mathbb{H}) \longrightarrow K\left((E / H)^{(n)}\right)
$$

by $\alpha^{\prime}=j^{*} \circ \alpha$. Then we have

Lemma 2.1. The following diagram is commutative:

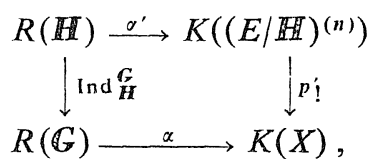

where Ind $\underset{\boldsymbol{H}}{\mathbf{G}}$ is the induction homomorphism defined by Segal [16] (see also [10]).

Proof. This is an easy consequence of the commutative diagram

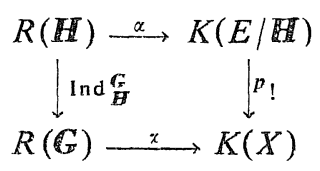

which is Proposition 5.4 of Nishida [14].

Let $\widetilde{S p h} *($ ) be the generalized cohomology theory defined by the stable spherical fibrations and $\operatorname{Sph}(X)=\widetilde{\operatorname{Sph}^{0}}\left(X_{+}\right)$. Define 


$$
p_{*}^{\prime}: K\left((E / \boldsymbol{H})^{(n)}\right) \longrightarrow K(X)
$$

and

$$
p_{*}^{\prime}: \operatorname{Sph}\left((E / \boldsymbol{H})^{(n)}\right) \longrightarrow \operatorname{Sph}(X)
$$

by a similar way to $p_{!}^{\prime}$ using the suspension isomorphisms defined by the infinite loop space structures defined by the $\Gamma$-structures (cf. Segal [17]). Since $J$ is an infinite loop map with respect to these infinite loop space structures, we have (cf. Nishida [14]).

Lemma 2.2. The following diagram is commutative:

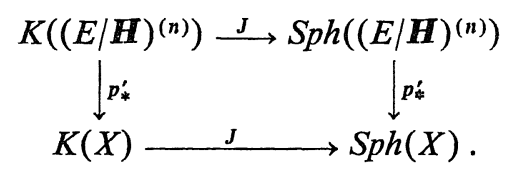

By May [13], the infinite loop space structure of $B \boldsymbol{U} \times \boldsymbol{Z}$ defined by the $\Gamma$-structure is equivalent to that defined by the Bott periodicity theorem. Then $p_{1}^{\prime}=p_{*}^{\prime}$ and so we have

Theorem 2.3. The diagram

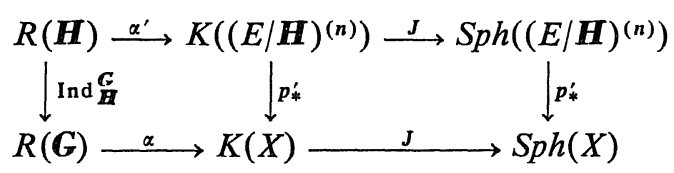

is commutative.

Quite similarly we have (cf. Hashimoto [10])

Theorem 2.4. The diagram

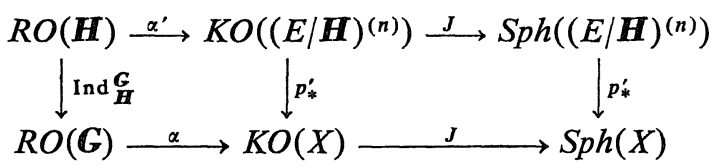

is commutative where $\operatorname{Ind}_{\boldsymbol{H}}^{\boldsymbol{G}}$ is the induction homomorphism of real representation rings defined by Hashimoto [10].

\section{§3. Proof of Theorem 1}

First recall the following lemmas.

Lemma 3.1. Let $f: Y \rightarrow Y^{\prime}$ be a (continuous) map and $y \in K\left(Y^{\prime}\right)$. If 
$k^{e} J \circ\left(\psi^{k}-1\right)(y)=0$, then $k^{e} J \circ\left(\psi^{k}-1\right)\left(f^{*}(y)\right)=0$.

Proof. This is an easy consequence of the following commutative diagram:

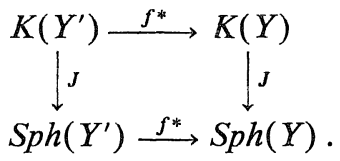

Lemma 3.2. For any complex line bundle $x$ over an $n$-dimensional CW complex $X$,

$$
k^{e(n \cdot k)} J \circ\left(y^{k}-1\right)(x)=0 .
$$

Proof. Since $x=f^{*}\left(\eta_{[n / 2]}\right)$ for some $f: X \rightarrow C P^{[n / 2]}$, this lemma follows immediately from Lemma 3.1.

To prove Theorem 1, we may assume that $X$ is a finite cell complex by Lemma 3.1, since $B \boldsymbol{U} \times \mathbb{Z}$ is skeleton finite (under a suitable cellular decomposition). So from now on $X$ is an $n$-dimensional finite cell complex.

For any $x \in K(X)$ we may assume that $x$ is an $m$-dimensional complex vector bundle for some $m$. Let $E$ be the total space of the associated principal $\mathbb{U}(m)$-bundle. Let

$$
\beta_{m}: \mathbb{U}(1) \times \mathbb{U}(m-1) \longrightarrow \mathbb{U}(1)
$$

be the first projection and

$$
c_{m}: \mathbb{U}(m) \longrightarrow \mathbb{U}(m)
$$

be the identity map. Put $\mathbb{G}=\mathbb{U}(m)$ and $\mathbb{H}=\mathbb{U}(1) \times \mathbb{U}(m-1) \subset \mathbb{U}(m)$. The following is due to [11] (see also Appendix):

Lemma 3.3. $\operatorname{Ind}_{\boldsymbol{H}}^{\boldsymbol{C}}\left(\beta_{m}\right)=\iota_{m}$

Note that $\alpha\left(c_{m}\right)=x$. Since $\mathbb{G}$ is connected we have

Lemma 3.4. For any integer $k, \psi^{k} \circ \operatorname{Ind}_{H}^{G}=\operatorname{Ind}_{H}^{G} \circ \psi^{k}$.

A proof is given in [12].

Now we can prove Theorem 1. Note that $\alpha \circ \psi^{k}=\psi^{k} \circ \alpha$ and $\alpha^{\prime} \circ \psi^{k}=\psi^{k} \circ \alpha^{\prime}$ by definitions and

$$
\begin{aligned}
& J \circ\left(\psi^{k}-1\right)(x)=J \circ\left(\psi^{k}-1\right)\left(\alpha\left(\iota_{m}\right)\right) \\
& =J \circ\left(\psi^{k}-1\right)\left(\alpha\left(\operatorname{Ind}_{\boldsymbol{H}}^{\boldsymbol{G}}\left(\beta_{m}\right)\right)\right) \quad \text { (by Lemma 3.3) } \\
& =J \circ \alpha \circ \operatorname{Ind}_{\boldsymbol{H}}^{\boldsymbol{G}} \circ\left(\psi^{k}-1\right)\left(\beta_{m}\right) \quad \text { (by Lemma 3.4) }
\end{aligned}
$$




$$
\begin{aligned}
& =p_{*}^{\prime} \circ J \circ \alpha^{\prime} \circ\left(\psi^{k}-1\right)\left(\beta_{m}\right) \quad \text { (by Theorem 2.3) } \\
& =p_{*}^{\prime} \circ{ }^{\circ} \circ\left(\psi^{k}-1\right) \circ \alpha^{\prime}\left(\beta_{m}\right) .
\end{aligned}
$$

Since $\alpha^{\prime}\left(\beta_{m}\right)$ is a complex line bundle over an $n$-dimensional finite cell complex $(E / \boldsymbol{H})^{(n)}$,

$$
k^{e(n, k)} J \circ\left(\psi^{k}-1\right)\left(\alpha^{\prime}\left(\beta_{m}\right)\right)=0
$$

by Lemma 3.2. So

$$
k^{e(n, k)} J \circ\left(\psi^{k}-1\right)(x)=k^{e(n, k)} p_{*}^{\prime} \circ J \circ\left(\psi^{k}-1\right)\left(\alpha^{\prime}\left(\beta_{m}\right)\right)=0 .
$$

This completes the proof.

\section{§4. Proof of Theorem 2}

Let $r: K(X) \rightarrow K O(X)$ be the realization homomorphism defined by forgetting complex structures. Then the following lemmas are well known:

Lemma 4.1. $2 K O(X) \subset \operatorname{Im} r$.

Lemma 4.2. The diagram

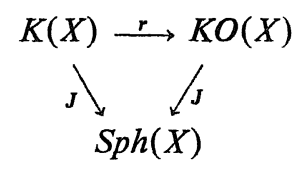

is commutative.

If $k$ is even, then $k x \in \operatorname{Im} r$ for any $x \in K O(X)$. So $k^{e^{\prime}(n, k)} J \circ\left(\psi^{k}-1\right)(x)$ $=k^{e(n, k)} J \circ\left(\psi^{k}-1\right)(k x)=0$ by Theorem 1 .

From now on $k$ is an odd integer. First we prove

Lemma 4.3. If $X$ is an n-dimensional $C W$ complex and $x \in K O(X)$ is a linear combination of one or two dimensional real vector bundles, then

$$
k^{e(n, k)} J \circ\left(\psi^{k}-1\right)(x)=0 .
$$

Proof. By Theorem 1, Lemma 4.1 and Lemma 4.2,

$$
2 k^{e(n, k)} J \circ\left(\psi^{k}-1\right)(x)=k^{e(n, k)} J \circ\left(\psi^{k}-1\right)(2 x)=0 .
$$

But by the Adams conjecture for one or two dimensional real vector bundles [2], $J \circ\left(\psi^{k}-1\right)(x)$ is an odd torsion. This completes the proof. Q.E.D.

Lemma 4.4. Let $\boldsymbol{G}$ be a compact Lie group and $\boldsymbol{H}$ be its closed subgroup. 
If $\left(\left|\boldsymbol{G} / G^{0}\right|, k\right)=1$ ( $\boldsymbol{G}^{0}$ denotes the connected component of the identity), then

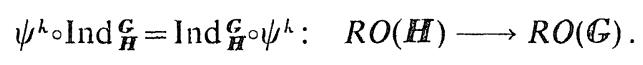

A proof is given in Appendix.

In particular we have

Corollary 4.5. If $\boldsymbol{G}=\mathscr{O}(2 n+1)$ and $\mathbb{H}=\mathscr{O}(2) \times \mathscr{O}(2 n-1) \subset \mathcal{O}(2 n+1)$, then $\psi^{k} \circ \operatorname{Ind}_{H}^{G}=\operatorname{Ind}{ }_{H}^{G} \circ \psi^{k}$ for any' odd integer $k$.

Let $c$ be the identity of $G, v: H \rightarrow O(2)$ be the first projection and $\mu$ : $\boldsymbol{G} \rightarrow \boldsymbol{O}(1)$ be the determinant (cf. Hashimoto [10]). Then the following is Proposition 5 of [10]:

Lemma 4.6. $\quad c=\operatorname{Ind}_{H}^{G}(v)+\mu$.

Now using Lemma 4.3, Lemma 4.6 and Theorem 2.4 instead of Lemma 3.2, Lemma 3.3 and Theorem 2.3 respectively, we can prove Theorem 2 by a similar way.

Remark 4.7. We can prove Theorem 1 of Sigrist and Suter [18] by making use of Theorem 2.4 and Lemma 4.6. In the proof of [18], the fact that $s$-map induces a homomorphism of $J^{\prime \prime}([2])$ is not clear, since $s$-map does not commute with the Adams operations. Moreover the Atiyah transfer does not commute with the Adams operations. The fact that the Atiyah transfer coincides with the Becker-Gottlieb transfer, which is an easy consequence of the AtiyahSinger index theorem for elliptic families ([6]), seems to be necessary.

\section{Appendix}

Let $\boldsymbol{G}$ be a compact Real Lie group and $R R(\mathbb{G})$ be the Real representation ring. If we forget involutions, a homomorphism $r: R R(\boldsymbol{G}) \rightarrow R(\boldsymbol{G})$ is defined. As is well known $r$ is a monomorphism (cf. Atiyah-Segal [5]). Moreover we know the diagram

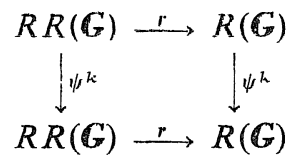

is commutative. Let $\boldsymbol{H}$ be a Real subgroup of $\mathbb{G}$ and $\operatorname{Ind}_{\boldsymbol{H}}^{\boldsymbol{G}}$ be the induction homomorphism defined by Hashimoto [10]. Then the diagram 


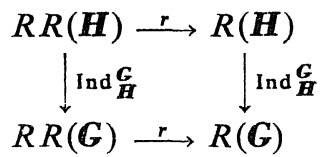

is commutative (cf. [10]). Now applying Theorem 1 of [12], we have

Lemma A.1. If $\left(\left|\boldsymbol{G} / \boldsymbol{G}^{0}\right|, k\right)=1$, then

$$
\psi^{k} \circ \operatorname{Ind}_{\boldsymbol{H}}^{\boldsymbol{G}}=\operatorname{Ind}_{\boldsymbol{H}}^{\boldsymbol{G}} \psi^{k}: R R(\boldsymbol{H}) \longrightarrow R R(\boldsymbol{G}) .
$$

If the involution of $\boldsymbol{G}$ is trivial, then $R R(\boldsymbol{G})=R O(\boldsymbol{G})$ and $\psi^{k}$ and $\operatorname{Ind}_{\boldsymbol{H}}^{\boldsymbol{G}}$ on $R O$ ( ) coincide with those on $R R($ ). So Lemma 4.4 is proved.

\section{References}

[1] Adams, J. F., Vector fields on spheres, Ann Math., 75 (1962), 603-632.

[2] — On the groups $J(X)$ I, Topology, 2 (1963), 181-195.

[3] Lecture on Lie groups, Benjamin, 1969.

[4] Atiyah, M. F., K-theory, Benjamin, 1967.

[5] Atiyah, M. F, and Segal, G. B., Equivariant $K$-theory and completions, J. Differential Geometry, 3 (1969), 1-18.

[6] Atiyah, M. F. and Singer, I. M., The index of elliptic operators IV, Ann. Math., 93 (1971), 119-138.

[7] Becker, J. C., Characteristic classes and $K$-theory, Lecture Notes in Math., 428, Springer, 132-143.

[ 8 ] Becker, J. C. and Gottlieb, G. H., The transfer maps and fibre bundles, Topology, 14 (1975), 1-12.

[9] Friedlander, E., Fibrations in etale homotopy theory, Publ. I.H.E.S., 42 (1972), 281-322.

[10] Hashimoto, S., The transfer map in $K R_{G}$-theory (to appear in Osaka $J$. Math.).

[11] Kono, A., Segal-Becker theorem for KR-theory, Japan J. Math., 7 (1981), 195-199.

[12] — Induced representations of compact Lie groups and the Adams operations, Publ. RIMS, Kyoto Univ., 17 (1981), 553-556.

[13] May, J. P., $E_{\infty}$ ring spaces and $E_{\infty}$ ring spectra, Lecture Notes in Math., 577, Springer.

[14] Nishida. G. The tranfer homomorphism in equivariant generalized cohomology theories, J. Math. Kyoto Univ., 18 (1978), 435-451.

[15] Quillen, D., The Adams conjecture, Topology, 10 (1971), 67-80.

[16] Segal, G. B., The representation ring of a compact Lie group, Publ. I.H.E.S., 34 (1968), 113-128.

[17] — Categories and cohomology theories, Topology, 13 (1974), 293-312.

[18] Sigrist, F. and Suter, U., On the exponent and the order of the group $\tilde{J}(X)$, Lecture Notes in Math., 673, Springer, 116-122.

[19] Sullivan, D., Genetics of homotopy theory and the Adams conjecture, Ann. Math., 100 (1974), 1-79. 